\title{
EchoGéo
}

$7 \mid 2008$

Les nouveaux enjeux régionaux dans l'océan Indien occidental

\section{Entretien avec Nadia Belaïdi}

La lutte contre les atteintes globales à l'environnement : vers un ordre public écologique ? Editions Bruylant, Bruxelles, 2008, 498 pages, (ISBN 978-2-8027-2470-4)

\section{Frédéric Ogé}

\section{(2) OpenEdition}

12 Journals

\section{Édition électronique}

URL : https://journals.openedition.org/echogeo/9933

DOI : $10.4000 /$ echogeo.9933

ISSN : 1963-1197

Éditeur

Pôle de recherche pour l'organisation et la diffusion de l'information géographique (CNRS UMR 8586)

Référence électronique

Frédéric Ogé, «Entretien avec Nadia Belaïdi », EchoGéo [En ligne], 7 | 2008, mis en ligne le 04 février 2009, consulté le 31 juillet 2021. URL : http://journals.openedition.org/echogeo/9933 ; DOI : https:// doi.org/10.4000/echogeo.9933

Ce document a été généré automatiquement le 31 juillet 2021.

EchoGéo est mis à disposition selon les termes de la licence Creative Commons Attribution - Pas d'Utilisation Commerciale - Pas de Modification 4.0 International (CC BY-NC-ND) 


\section{Entretien avec Nadia Belaïdi}

La lutte contre les atteintes globales à l'environnement : vers un ordre public écologique ? Editions Bruylant, Bruxelles, 2008, 498 pages, (ISBN

978-2-8027-2470-4)

\section{Frédéric Ogé}

$1 \quad$ Les éditions Bruylant sont bien connues pour leur sérieux et la qualité de leurs choix éditoriaux. Leur décision de publier un ouvrage aussi important que l'opus tiré de la thèse de doctorat de Nadia Belaïdi n'est donc pas anodine. En effet, ce beau livre est loin de ne concerner que les seuls juristes : il peut, il doit être mis entre les mains des géographes, des politologues, des sociologues mais aussi des écologues dans les facultés de sciences, des élèves ingénieurs des Ecoles des Mines (par exemple). Il est, plus largement, fort utile pour tous les acteurs de l'aménagement du territoire qu'ils interviennent dans la sphère de la décision politique, dans le champ économique ou qu'ils animent des structures visant à protéger notre environnement. Pourquoi ? Parce qu'il oblige tout lecteur à réfléchir, à s'interroger, à remettre en perspective un certain nombre de concepts considérés comme majeurs en ce début de vingt-et-unième siècle. La tâche n'est pas aisée : Nadia Belaïdi ne nous offre pas un vade-mecum du droit de l'environnement. Elle tente simplement d'établir des constats et de proposer des pistes. Son livre suscitera certainement des réactions négatives ou frileuses : rien d'étonnant à cela puisqu'il ne peut laisser indifférent, nous contraignant à dépasser les exposés méthodologiques «classiques » sans tomber dans le piège de l'incantation pseudo épistémologique.

2 A cette fin, l'ouvrage - d'une lecture relativement aisée quand bien même le lecteur n'a pas passé plusieurs années sur les bancs des facultés de droit et craindrait de se perdre face à des références jurisprudentielles et réglementaires nombreuses et indispensables - nous entraine sur trois terrains. Dans une première partie (p. 22-128) il est affirmé que les catégories juridiques classiques ne pourraient être des outils vraiment adéquats pour qualifier et quantifier les atteintes globales à l'environnement (définies comme tout facteur anthropique pouvant perturber le système terre préalablement appréhendé comme une combinaison d'équilibres et interdépendances indispensables au maintien des supports de toute vie). Ni le droit civil ni le droit pénal ne 
parviendraient à prévenir et réparer totalement dommages et nuisances. Cela expliquerait l'émergence progressive d'un droit spécifique: le droit de l'environnement. Mais ce dernier serait beaucoup trop limité actuellement. C'est ce qu'entend démontrer Nadia Belaïdi dans la deuxième partie de son livre (p. 129-284). Certes, elle se veut relativement optimiste en soulignant l'importance de la mise en place de ce que certains définissent comme une branche de la science juridique méritant plus d'intérêt que le droit civil (par exemple), en mettant en exergue le "supplément d'âme " qu'impliquerait le droit à l'environnement (qui plus est "de qualité »), en analysant l'émergence de principes tels que le principe pollueur-payeur, le principe de prévention, le principe de précaution. Mais elle a également conscience du fossé encore profond entre les impératifs de la raison d'Etat (ne serait-ce que «l'obligation» de croissance du P.N.B.) et la recherche de l'intérêt commun, entendu comme intérêt intergénérationnel de l'espèce humaine, solidarité entre territoires considérés comme plus ou moins "développés", perception de la nécessité de maintenir micro et macro écosystèmes de plus en plus fragilisés. C'est donc fort logiquement qu'elle intitule sa troisième et dernière partie (p. 285-461) : «Des atteintes globales à l'environnement révélatrices de la nécessité de construire un 'ordre public écologique' ». Après y avoir comparé les démarches occidentales et non-occidentales en matière de protection de l'environnement en focalisant notre réflexion sur les concepts de temporalité et de transmissibilité, elle affirme la nécessité d'une régulation de nos comportements en posant que la protection des droits fondamentaux liés à un environnement de qualité ne peut être réalisée qu'en construisant un nouvel ordre public (local ou/et global, peu importe la dimension du territoire considéré). Cela implique de repenser nos valeurs fondamentales, redéfinir en quelque sorte les priorités de notre société. Nadia Belaïdi reste réaliste : «Beaucoup de chemin reste à parcourir » écrit-elle (page 468). Mais l'optimisme habite sa plume. A l'en croire, un nouveau principe de gouvernement devrait s'imposer progressivement: le concept de développement durable. En son nom, nous revisiterons les normes fondamentales régissant les liens (au sens de Michel Serres) entre humains mais aussi avec toutes les composantes des écosystèmes. Certains estimeront sans doute que ces pages n'énoncent qu'un rêve. D'autres affirmeront que l'actualité récente a prouvé qu'un rêve peut devenir réalité. Toujours est-il que l'ouvrage publié par Bruylant nous contraint justement à prendre position par rapport à la thèse proposée par Nadia Belaïdi. $\mathrm{Ne}$ serait-ce que pour cette raison il faut le lire d'autant plus que cet exercice est fort aisé du fait d'une écriture claire et d'une bonne qualité typographique.

EchoGéo

4 En quoi ce livre peut-il être considéré comme «novateur" ou «dérangeant " par rapport aux autres publications disponibles actuellement sur le marché et traitant du droit de l'environnement?

$5 \quad$ Nadia Belaïdi

6 L'aspect novateur de l'ouvrage réside en ce qu'il propose non pas une lecture de la réglementation en matière d'environnement mais une appréciation de son adéquation aux problématiques qu'elle est censée saisir. Il ne s'agit donc pas de lire et/ou d'analyser la règle mais d'en identifier les fondements pour mettre en relief la relation entre la réalité des problématiques environnementales retenues dans le cadre de cette étude (changement climatique principalement) et les objectifs poursuivis par le droit 
de l'environnement. Plus qu'à la lettre, on s'intéresse à son esprit afin d'en analyser sa pratique.

7 EchoGéo

8 Ne peut-on craindre que ce livre contribue à accentuer la confusion entre l'affirmation d'une éthique et l'édiction de règles, de normes ainsi que leur interprétation? de ne pas faire fausse route dans la mesure où la globalisation viserait à réduite justement un encadrement réglementaire synonyme de carcan freinant tout progrès, toute amélioration de la condition humaine?

$$
\begin{aligned}
& \text { Néanmoins, vous semblez y croire : est-ce une « figure de style } \\
& \text { du temps? Si non, quelle place les juristes pourraient-ils tenir? }
\end{aligned}
$$

La confusion est entretenue lorsque l'on ne fait pas l'effort de dissocier les interférences entre le droit, la politique et l'économie. Positionner le droit sur ses bases - régir la vie en société sur la base des valeurs portées par cette société - permet précisément d'éviter les confusions et mélanges des genres précités.

\section{EchoGéo}

Appeler à un "changement de logique" est-ce le rôle du juriste ou, plus largement, du chercheur en sciences humaines et sociales ? N'est-ce pas plutôt du ressort du moraliste?

Nadia Belaïdi

L'appel au «changement de logique » ressort de l'étude. Envisager le droit sous l'angle de la protection de l'environnement - compris en tant que milieu de vie - aboutit à considérer que la logique actuelle de construction et de mise en application du droit de l'environnement manque l'objectif poursuivi. C'est donc simplement en tant que chercheure en droit international de l'environnement que j'aboutis à cette considération.

EchoGéo

Nadia Belaïdi

L'ordre public écologique est fondé sur la promotion des valeurs sociales essentielles communes à tous les peuples dans leur diversité. Il est l'antinomie du mouvement d'uniformisation que tend à devenir le phénomène de mondialisation. Il s'agit avec l'ordre public écologique non pas d'imposer des manières de voir ou de faire mais de positionner les valeurs sociales propre à favoriser la vie dans sa diversité comme une référence dans le cadre de laquelle les Etats et les peuples sont libres d'agir selon leur perception et leur pratique et à leur propre rythme. Cette posture est bien différente de l'exportation des modèles économique et politique dominants que prône aujourd'hui le phénomène de mondialisation.

\section{EchoGéo}

La pluridisciplinarité est loin d'être aisée à pratiquer, tout le monde en convient. Néanmoins, vous semblez y croire : est-ce une « figure de style » pour répondre à l'air

\section{Nadia Belaïdi}

Pour traiter des questions aussi complexes que celles relatives à l'environnement, le recours à la pluridisciplinarité me parait indispensable et le «juriste» comme tout autre spécialiste des sciences humaines et sociales y a sa place. Elle est même décisive 
dans la mesure où il est celui qui est apte à traduire les apports des autres sciences en des termes qui, repris dans la règle, seront susceptible d'avoir un impact sur la société. Réciproquement, il est celui qui, par la lecture des textes qu'il effectue, peut identifier la « vision du monde » qui s'affirme et ainsi permettre les comparaisons dans le temps, signaler les incohérences avec les réalités grâce aux analyses et apports des autres disciplines. Cette complémentarité entre les disciplines permet alors une analyse précise des problématiques environnementales dans leur composante écologique, sociale, culturelle et économique.

EchoGéo

Certains soulignent combien difficile est la « simple » mise en œuvre des principes-clés à la base d'une politique environnementale au «simple " niveau national. Parler de construire un droit à portée planétaire, n'est-ce pas faire preuve d'un total irréalisme?

\section{Nadia Belaïdi}

Dans la mesure où il s'agit d'un droit à portée planétaire et non d'une réglementation, il me semble des plus réalistes d'envisager les valeurs sociales communes qui permettent de dépasser les conflits parce que l'on parle de l'intérêt commun. Cela est possible dès lors que l'on modifie les champs spatial et temporel dans lesquels on appréhende le monde et nos actions. C'est précisément ce que propose le droit international. Son rôle est de tendre vers un idéal qui n'existe pas encore. Compte-tenu de tout ce qui a été évoqué précédemment, il est vraisemblable qu'il faille tendre vers quelques utopies pour réaliser un minimum d'avancées. Or le droit international est précisément, comme l'a souligné le Professeur René-Jean Dupuy en 1984 (RCADI p. 503), un droit utopique au sens élevé du terme.

\section{EchoGéo}

Après avoir brossé un état des lieux assez réaliste et par conséquent relativement pessimiste, vous développez des propositions en matière de lutte contre les atteintes globales à l'environnement que certains définiront comme utopistes ou trop optimistes. Est-ce votre rôle de chercheur de développer des mirages?

\section{Nadia Belaïdi}

C'est mon rôle de chercheur de douter que ce que l'on montre comme étant la réalité, la seule vérité et la seule voie possible le soit réellement. En tant que chercheure en droit, je peux interroger le sens des règles édictées pour le «bon fonctionnement de la société " et mettre en relief d'une part les hiatus entre la règle et les réalités écologiques et sociales et d'autre part des moyens de les éviter. Les perspectives avancées ne sont pas des mirages, elle relève de potentialités auxquelles il reste à donner forme et force juridique. 